\title{
Spectrum of Uropathogens and Antimicrobial Susceptibility in Clinically Diagnosed Cases of Urinary Tract Infection in the Bamenda Regional Hospital, Cameroon
}

\author{
Marie Ebob Agbortabot Bissong ${ }^{1,2, ~ *, ~ C a r l s o n ~ M b a h ~}{ }^{1}$, Frank Eric Tatsing Foka ${ }^{2}$, \\ Henri-Lucien Kamga ${ }^{1}$ \\ ${ }^{1}$ Department of Medical Laboratory Sciences, Faculty of Health Sciences, University of Bamenda, Bamenda, Cameroon \\ ${ }^{2}$ Department of Biological Sciences, Faculty of Agriculture, Science and Technology, North West University, Mafikeng, South Africa \\ Email address: \\ mabissong@yahoo.com (M. E. A. Bissong), mbahcarlson93@gmail.com (C. Mbah), frankeric2023@yahoo.fr (F. E. T. Foka), \\ henrikamga2002@yahoo.fr (H-L Kamga) \\ *Corresponding author
}

\section{To cite this article:}

Marie Ebob Agbortabot Bissong, Carlson Mbah, Frank Eric Tatsing Foka, Henri-Lucien Kamga. Spectrum of Uropathogens and Antimicrobial Susceptibility in Clinically Diagnosed Cases of Urinary Tract Infection in the Bamenda Regional Hospital, Cameroon. American Journal of Health Research. Vol. 5, No. 2, 2017, pp. 19-24. doi: 10.11648/j.ajhr.20170502.11

Received: December 8, 2016; Accepted: January 9, 2017; Published: March 2, 2017

\begin{abstract}
Urinary tract infections (UTIs) are among the common bacterial infections in humans both in the community and hospital settings. In spite of the availability and use of antimicrobial drugs, UTIs have shown increasing trends in recent years. Antimicrobial resistance among uropathogens is a serious health threat as this may complicate treatment, eventually resulting to more severe disease. Despite the importance of UTI, there is paucity of information on the aetiology of this disease especially in the Northwest Region. For that reason, the present study was designed with the aim to determine the spectrum of uropathogens and antimicrobial resistance of isolates in cases of symptomatic bacteriuria. To achieve this goal, midstream urine specimens were inoculated unto CLED media and isolates were identified using the Enterosystem 18R. Significant bacteriuria was considered with bacterial growth of $\geq 10^{5} \mathrm{CFU} / \mathrm{ml}$. Susceptibility testing was performed using the Kirby Bauer disk diffusion method and the accuracy of the dipstick test was determined using culture as a gold standard. Significant bacteriuria was observed in $50 \%$ of the participants and the predominant uropathogens isolated were P. aeruginosa (22.4\%), Klebsiella spp (14.3\%), S. aureus (14.3\%), CNS (14.3\%) and E. coli $(12.2 \%)$. High resistance (80-98\%) among uropathogens was observed against clindamycin, amoxicillin and doxycycline. For the dipstick test, leucocyte esterase and nitrite showed high sensitivity $(93.9 \%)$ and specificity $(100 \%)$ respectively, for detecting UTI. The results of this study highlights the importance of Pseudomonas aeruginosa in the aetiology of both community and hospital acquired UTIs. In addition, gentamicin is the most effective agent against uropathogens in the study area.
\end{abstract}

Keywords: UTI, Symptomatic Bacteriuria, Aetiology, Screening Methods, Antimicrobial Resistance, Cameroon

\section{Introduction}

Urinary tract infections (UTIs) are among the common bacterial infections in humans both in the community and hospital settings [1]. It is estimated that over $50 \%$ of women and over $13 \%$ of men specifically, will have a UTI at least once in their lifetime with $20 \%$ to $30 \%$ of women experiencing recurring infections [2,3]. Studies have shown that UTIs are responsible for a large proportion of antimicrobial consumption in and out of the hospital $[4,5,6]$. In spite of the availability and use of antimicrobial drugs, UTIs have shown increasing trends in recent years with much of the increase relating to antibiotic resistance in urinary tract pathogens [6, 7].

Escherichia coli has been reported to be the predominant causative agent of UTI $[3,6,7,8]$. However, recent reports have increasingly incriminated Staphylococci, Proteus 
mirabilis, Klebsiella pneumonia, Pseudomonas aeroginosa, Enterococci spp and Candida albicans in causing UTI [8, 9]. The gold standard for the diagnosis of UTI remains culture of non-contaminated urine $[5,10,11]$. Routine urine analysis is a very easy and quick test considered in the diagnosis of UTI especially when culture facilities are not available [11].

Antimicrobial resistance is a fundamental issue in the area of public health worldwide. Emerging resistant strains spread rapidly and threatens the ability to treat common infectious diseases; with severe outcome such as prolonged illness, disability and death [5]. There has been increasing reports of antibiotic resistance among uropathogens $[9,12]$.

In Cameroon, there are reports of high prevalence of asymptomatic bacteriuria $[9,12,13,14]$ but information on the aetiology of symptomatic bacteriuria is scarce especially in the Northwest Region. Based on this dearth, the present study was designed to determine the spectrum of uropathogens in clinically diagnosed cases of UTI in the Bamenda Regional Hospital (which serves majority of the population of the Northwest Region) and to determine the antimicrobial susceptibility pattern of isolates.

\section{Methodology}

\subsection{Study Location and Ethical Consideration}

This was a cross-sectional study conducted in the Microbiology Laboratory of the Bamenda Regional Hospital (BRH) from February to June 2016. BRH is a 400-bedded hospital with an intensive care unit (ICU), renal dialysis unit, gynaecology, diabetic unit, ANC unit and IWC unit. Ethical clearance for this study was obtained from the Northwest Regional Delegation for Public Health and the University of Bamenda.

\subsection{Study Population}

A total of 98 consented participants of all age groups, who presented at the Hospital with signs and symptoms of UTI (diagnosed by a competent Clinician) were recruited into the study. Participants who were currently on antibiotics or had taken antibiotics one week prior to the study were excluded.

\subsection{Sample Collection}

"Clean-catch" midstream urine was collected from each participant into sterile urine containers for analyses within 2 hours of collection.

\subsection{Laboratory Analyses}

Each sample was subjected to culture and dipstick test as described by previous reports [9, 12]. The samples were inoculated unto CLED agar (Laboratorios Conda, SA.) using $10 \mu 1$ wire loop and the plates incubated aerobically at $37^{\circ} \mathrm{C}$ for 24 hours. Urine specimens with bacterial growth of $\geq 10^{5}$ $\mathrm{CFU} / \mathrm{ml}$ were considered significant bacteriuria. Isolates with significant growth were identified by standard bacteriological methods [15]. Gram positive cocci were identified by the catalase and coagulase tests while Gram negative bacteria were identified using the Enterosystem 18R (BioMérieux, France).

The dipstick test was done using test strips (Condor-Teco, China) as described by the manufacturer. Antibiotic susceptibility testing of isolates was performed by the KirbyBauer disk diffusion technique using standard procedures [16]. The following antimicrobial agents were used: Gentamicin (10mcg), Doxycycline (30mcg), Ceftriaxone (30mcg), Ciprofloxacin (30 mcg), Clindamycin (20mcg), and Amoxicillin (30mcg) (Liofilchem, Italy) and the procedures were done according to standards [17].

\subsection{Statistical Analysis}

Data were analysed using the Statistical software Package for Social Sciences (SPSS) version 20.0. Significant differences between categorical variables were determined using the Chi-Square test at a $95 \%$ confidence level. $P<0.05$ was considered statistically significant.

\section{Results}

More women participated in the study than men $(74.5 \%$ and $25.5 \%$, respectively). The majority $(39.8 \%)$ of participants were in the age group of 16-25 years while no participant was less than 15 years. Bacteria were isolated from all the urine samples but out of the 98 urine samples, $49(50 \%)$ had significant bacteriuria. Significant bacteriuria was detected more $(52.0 \%)$ in males than in females $(49.3 \%)$. Meanwhile, participants above 45 years showed the highest prevalence (66-70\%) of bacteriuria. Although there was no significant difference, significant bacteriuria was detected more in participants from outpatient than inpatient ward (table 1).

Table 1. Prevalence of significant bacteriuria based on age, gender and ward.

\begin{tabular}{|c|c|c|c|c|c|}
\hline Parameter & Category & Significant bacteriuria (\%) & Non-significant bacteriuria (\%) & Total $(\%)$ & P value \\
\hline \multirow{2}{*}{ Gender } & Males & $13(52.0)$ & $12(48.0)$ & $25(25.5)$ & \multirow{2}{*}{0.815} \\
\hline & Females & $36(49.3)$ & $37(50.7)$ & $73(74.5)$ & \\
\hline \multirow{4}{*}{ Age (years) } & $\leq 15$ & $0(0.0)$ & $0(0.0)$ & $0(0.0)$ & \multirow{4}{*}{0.138} \\
\hline & $16-25$ & $18(46.2)$ & $21(53.8)$ & $39(39.8)$ & \\
\hline & $26-45$ & $12(38.7)$ & $19(61.3)$ & 31 (31.6) & \\
\hline & $46-60$ & $12(66.7)$ & $8(33.3)$ & $18(18.4)$ & \\
\hline \multirow{2}{*}{ Ward } & Inpatient & $17(54.8)$ & $14(45.2)$ & 31 (31.6) & \multirow{2}{*}{0.515} \\
\hline & Outpatient & $32(47.8)$ & $35(52.2)$ & $67(68.4)$ & \\
\hline
\end{tabular}


A total of 8 bacterial genera were isolated and the majority (71.4\%) belongs to the family Enterobacteriaceae (table 2). There was a significant difference in the distribution of uropathogens among study participants $(p=0.001)$. The most prevalent uropathogens were $P$. aeruginosa (22.5\%),
Klebsiella spp (14.3\%), S. aureus (14.3\%), C. N. S (14.3\%), E. coli (12.2\%), and Enterobacter spp (10.2\%). Uropathogens were isolated more $(32 / 49,65.3 \%)$ from outpatients than inpatients $(17 / 49,34.7 \%)$, although this difference was not statistically significant $(p=0.153)$.

Table 2. Distribution of uropathogens amongst study participants.

\begin{tabular}{|c|c|c|c|c|}
\hline Microorganism & Inpatients N (\%) & Outpatients N (\%) & Total N (\%) & P-value \\
\hline Pseudomonas aeruginosa & $3(17.6)$ & $8(25.0)$ & $11(22.5)$ & \\
\hline Escherichia coli & $2(11.8)$ & $4(12.5)$ & $6(12.2)$ & \\
\hline Klebsiella spp & $3(17.6)$ & $4(12.5)$ & $7(14.3)$ & \\
\hline Enterobacter spp & $2(11.8)$ & $3(9.4)$ & $5(10.2)$ & \\
\hline Serratia liquefaciens & $2(11.8)$ & $0(0.0)$ & $2(4.1)$ & 0.153 \\
\hline Salmonella arizonae & $2(11.8)$ & $0(0.0)$ & $2(4.1)$ & \\
\hline Citrobacter freundii & $1(5.8)$ & $0(0.0)$ & $1(2.0)$ & \\
\hline Staphylococcus aureus & $0(0.0)$ & $7(21.9)$ & $7(14.3)$ & \\
\hline CNS & $2(11.8)$ & $5(15.6)$ & $7(14.3)$ & \\
\hline Total & $17(100)$ & $32(100)$ & $49(100)$ & \\
\hline
\end{tabular}

$\stackrel{x}{\mathrm{P}}=0.001$ (Variables were compared across the column)

The susceptibility of isolates was tested against six different antimicrobial agents (table 3). Isolates showed the highest resistance against clindamycin $(98 \%)$ followed by amoxicillin $(89.8 \%)$ and doxycycline $(83.7 \%)$, while the lowest resistance was observed against gentamicin $(16.3 \%)$ (Table 3). None of the P. aeruginosa isolates was resistant to gentamicin. Gentamicin resistance was more common among isolates from in-patients than those from the out-patients $(\mathrm{P}=0.001)$ (Table 3).
Figure 1 shows the resistance patterns of the various bacterial isolates. All E. coli isolates were resistant to ceftriaxone, amoxicillin and clindamycin while all Enterobacter species were resistant to doxycycline, amoxicillin and clindamycin. None of the P. aeruginosa isolates was resistant to gentamicin and the lowest resistance to amoxicillin was exhibited by CNS.

Table 3. Distribution of antimicrobial resistance amongst inpatients and outpatients.

\begin{tabular}{lllll}
\hline Antibiotics & Inpatients (\%) & Outpatients (\%) & Total N (\%) \\
\hline Doxycycline & $14(34.1)$ & $27(65.9)$ & $41(83.7)$ & P value \\
Ciprofloxacin & $7(30.4)$ & $16(69.6)$ & $23(46.9)$ & 0.082 \\
Ceftriaxone & $11(40.7)$ & $16(59.3)$ & $27(55.1)$ & 0.732 \\
Gentamicin & $7(87.5)$ & $1(12.5)$ & $8(16.3)$ & 0.057 \\
Amoxicillin & $16(36.4)$ & $28(63.6)$ & $44(89.8)$ & 0.001 \\
Clindamycin & $17(35.4)$ & $31(64.6)$ & $48(98)$ & 0.687 \\
\hline
\end{tabular}

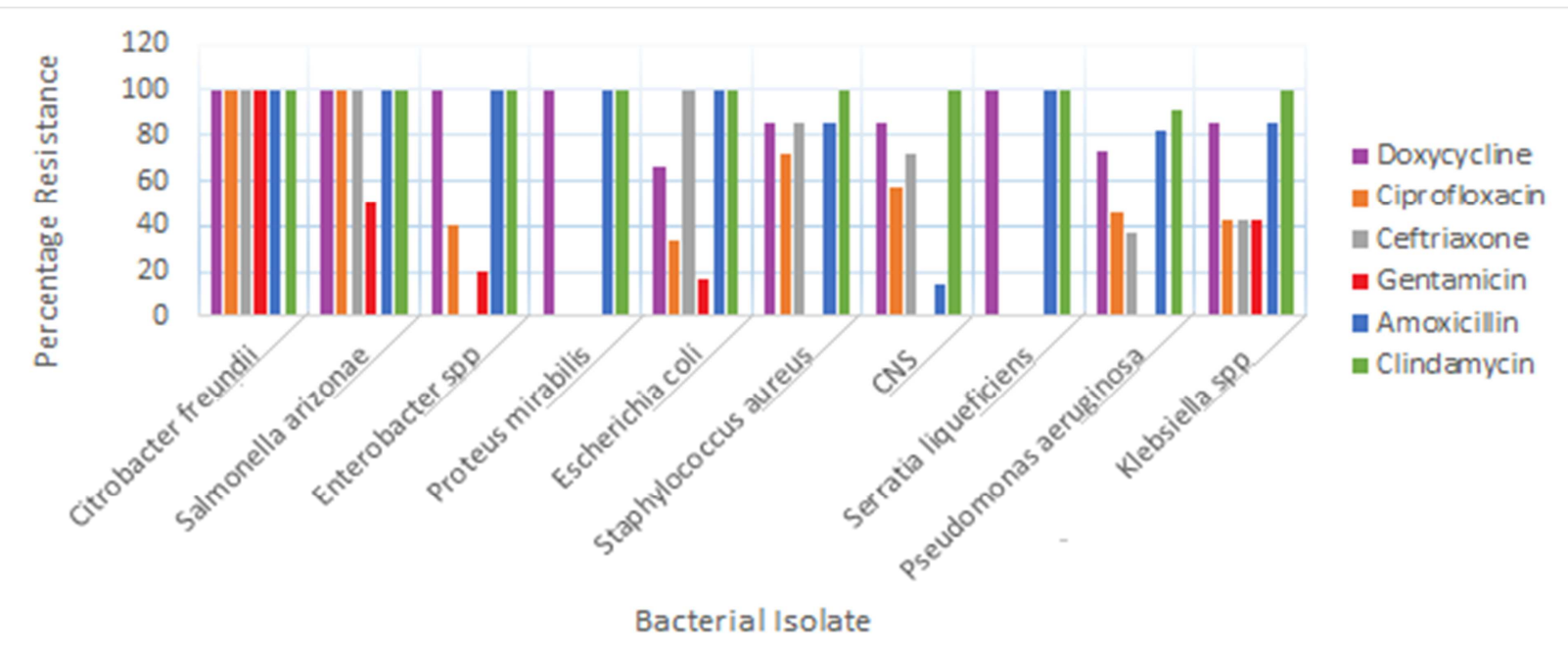

Figure 1. Antibiotic resistance patterns of bacterial isolates. 
The sensitivities and specificities of the infection-related parameters of the urinalysis test (leukocyte esterase, nitrite and red blood cells) using culture as gold standard are revealed on table 4 . The presence of nitrite, red blood cells and leucocyte esterase tests had sensitivities of $17.1 \%$, $55.1 \%, 93.9 \%$ and specificities of $100 \%, 79.6 \%$ and $67.4 \%$ respectively.

Table 4. Sensitivity and specificity of some dipstick test parameters using culture as gold standard.

\begin{tabular}{|c|c|c|c|c|c|c|}
\hline \multirow{2}{*}{ Characteristic } & \multicolumn{2}{|c|}{ Culture positive (Significant bacteriuria) } & \multicolumn{2}{|c|}{ Culture negative (Non-significant bacteriuria) } & \multirow{2}{*}{ Total } & \multirow{2}{*}{ P-value } \\
\hline & Positive & Negative & Positive & Negative & & \\
\hline Red blood cells & $27(55.1)$ & $22(44.9)$ & $10(20.4)$ & $39(79.6)$ & 98 & 0.004 \\
\hline Leukocyte esterase & $46(93.9)$ & $34(69.4)$ & $16(32.7)$ & $33(67.3)$ & 98 & 0.000 \\
\hline Nitrite & $15(30.6)$ & $34(69.4)$ & $0(0.0)$ & $49(100)$ & 98 & 0.000 \\
\hline
\end{tabular}

Sensitivity: Red blood cell (55.1\%), leucocyte esterase (93.9\%) and nitrite (30.6\%)

Specificity: Red blood cell (79.6\%), leucocyte esterase (67.4\%) and nitrite (100\%)

\section{Discussion}

This study was conducted to determine the spectrum of uropathogens in clinically diagnosed cases of UTI. Urinary tract infection is common worldwide and factors such as age, gender and diseases such as diabetes predispose to it. In the present study, there were more women than men $(74.5 \%$ and $25.5 \%$, respectively) and the majority $(39,39.8 \%)$ of the participants were aged 16 - 25years. It is worthy to note that the present study was conducted on individuals presenting with clinical signs and symptoms of UTI. This factor might be responsible for difference in participation observed with respect to gender and age.

Significant bacteriuria was detected in 49 out of $98(50 \%)$ of patients who presented with urinary symptoms at the hospital. These results are commensurate to previous reports in the country $[12,20]$ as well as in other countries [7]. Some previous studies $[9,13,14]$ in Cameroon have reported a lower prevalence of bacteriuria compared with the current study. This difference in the prevalence may be explained by the fact that most of these previous studies detected asymptomatic bacteriuria while the present study was focused on individuals presenting with clinical manifestation of UTI. It has been reported that bacteriuria or UTI is more common than in females than males $[7,9,12]$ perhaps due to anatomical differences. However, results of the present study did not show any significant difference $(\mathrm{P}>0.05)$ in the isolation of uropathogens between male and female (Table 1). This variation may be due differences in sample size; our sample size (98) was smaller than others [9, 12]. Although there was no significant difference, the prevalence of significant bacteriuria was higher in participants older than 45years. Similar results have been reported in which majority of cases of both complicated and uncomplicated UTI belonged to the elderly age group (50-79 years) [8]. The high rate of infections in the elderly may be due to a lower immune response.

The most common isolates from urine were $P$. aeruginosa (22.4\%), Klebsiella species (14.3\%), S. aureus (14.3\%), CNS (14.3\%) and E. coli $(12.2 \%)$ (Table 2). These results are commensurate with previous reports which revealed a change in the aetiology of both symptomatic and asymptomatic bacteriuria [6, 9]. In one of such studies, Bissong and colleagues reported the staphylococci as the predominant cause of asymptomatic bacteriuria in diabetic patients. Also the predominance of $P$. aeruginosa in this study may be explained by the fact that this bacterium is a nosocomial pathogen and some patients were hospitalized while majority of the outpatients had recently been hospitalized. A similar study [6] in Kuwait reveals that there is a difference in prevalence rate of $E$. coli $(12.2 \%)$, which indicates a decline in the proportion of patient with E. coli UTI. Klebsiella species are increasingly being incriminated in UTIs [7, 13]. The aetiological profile of UTI in the present study shows considerable shift from previous studies $[6,7,8,18,19]$. The variation in the aetiology of UTI could be attributed to differences in geographical location, screening methods, sample size, setting of study (Hospital versus community) and socioeconomic status [9, 13].

Antimicrobial susceptibility testing revealed that clindamycin showed the highest (98\%) resistance followed by amoxicillin $(89.8 \%)$ and doxycycline $(83.7 \%)$. High resistance of uropathogens to these antimicrobial agents has previously been reported $[3,9,12,20]$. Gram negative bacteria are generally resistant to clindamycin and resistance to this drug is usually as a result of drug inactivation, efflux pumps and or methylation of the target site [22, 23]. Resistance to amoxicillin is probably due to beta lactamase production [22]. The lowest resistance $(16.3 \%)$ was observed against gentamicin and resistance to this antibiotic was more common among isolates from in-patients than those from the out-patients $(p=0.001)$ (table 3$)$. It is possible that hospitalized patients might have been exposed more to gentamicin than the outpatients; however, the duration of hospitalization was not considered in the present study. Interestingly, none of the $P$. aeruginosa isolates was resistant to gentamicin. Furthermore, all E. coli isolates were resistant to ceftriaxone, amoxicillin and clindamycin while all Enterobacter species were resistant to doxycycline, amoxicillin and clindamycin (figure 1). Resistance of gram negative bacteria to cephalosporins and other cell wall antimicrobials are common $[24,25,26]$. Ciprofloxacin is a common drug in the treatment of UTI in our setup. Apart from Citrobacter and Salmonella species, gram positive 
bacteria showed a relatively higher resistance to ciprofloxacin compared with gram negative bacteria. It has been reported that gram positive bacteria are generally resistant against this antibiotic [27].

In testing for the accuracy of the dipstick test in the diagnosis of UTI, the highest sensitivity and specificity was noted for leucocyte esterase $(93.9 \%)$ and nitrite $(100 \%)$, respectively. However, none of these parameters singly was relatively accurate (high sensitivity and high specificity) for diagnosing UTI. Similar observations have been reported by other authors [11, 13]. In one of such studies, Ozturk et al reported a sensitivity and specificity of leucocyte esterase of $85 \%$ and $40 \%$, respectively and of nitrite of $61 \%$ and $99 \%$, respectively. These reports are indicative that a combination of these two parameters (leucocyte esterase and nitrite) is important for the accuracy of the dipstick test.

\section{Conclusion}

Results of this study revealed a change in the aetiology of UTI from $E$. coli dominance to $P$. aeruginosa and a high resistance $(80-98 \%)$ of uropathogens to commonly used antimicrobials. Thus, it is imperative for proper tests to be carried out prior to treatment, to determine the susceptibility of uropathogens. In addition, a combination of the urine leucocyte esterase and nitrite may be useful in the diagnosis of UTI in the absence of facilities for culture.

\section{References}

[1] K. Hryniewicz, K. Szczypa, A. Sulikowska, K. Jankowski, K. Betlejewska, W. Hryniewicz "Antibiotic susceptibility of bacterial strains isolated from urinary tract infections in Poland: Journal of Antimicrobial Chemotherapy" 2001, 47: 73-780.

[2] E. Kastanus, A. Kyriakides, K. Hadjigeorgiou, C. Pitris "A Novel Method For Bacteria UTI Diagnosis Using Raman Spectroscopy" International Journal of spectroscopy, 2012, 19: 1-13.

[3] M. Totsika, D. G. Moriel, A. Idris, B. A. Rogers, D. J. Wurpel, M. D. Phan, et al. "Uropathogenic Escherichia coli mediated urinary tract infection" Curr Drug Targets, 2012, 13 (11): 1386-99.

[4] R. Yakubov, V. D. M. Akker, K. Machamad, A. Hochberg, E. Nadir, A. Klein "Antimicrobial resistance among uropathogens that cause childhood community-acquired urinary tract infections in Central Israel" Pediatr Infect Dis J, 2016; 12 [Epub ahead of print].

[5] WHO. Antimicrobial resistance - World Health Organization 2016.

[6] www.who.int/mediacentre/factsheets/fs194/en/.

[7] N. A. Sweih, W. Jamal, V. O. Rotimi « Spectrum and Antimicrobial Resistance of Uropathogens Isolated from hospital and Community Patients with Urinary Tract Infection in two Large Hospital in Kuwait" Med Princpract, 2005; 14: 401-407.
[8] N. Suneetha, P. Subbulu, S. Usha, V. Rani, B. K. Reddy "Bacteriological Study of Urinary Tract Infection" International Journal of Interdisciplinary and Multidisciplinary Studies, 2015; 2: 11-16.

[9] M. Eshwarappa, R. Dosegowda, I. V. Apramega, M. W. Khan, P. S. Kumar, P. Kempegowda "Clinic-microbiological profile of urinary tract infection in south India: Indian Journal of Nephrology 2011; 21: 30-36.

[10] M. E. A. Bissong, P. N. Fon, F. O. Tabe-Besong, T. N. Akenji "Asymptomatic bacteriuria in diabetes mellitus patients in Southwest Cameroon" African Health Sciences, 2013, 13: 661-666.

[11] N. S. Hussein "Clinical, Etiology and Antibiotic Susceptibility Profiles of Community acquired Urinary Tract Infection in A Baghdad Hospital” Med Surg Urol, 2014, 3: 1-5.

[12] A. Ozturk, Z. Ginis, T. Hanci, Z. Yildiz, M. Y. Taslipinar, F. Ucar, G. Ozturk, A. Yalcindag, E. F. Alay, G. Erden "Evaluation of diagnostic performance of routine automated urinalysis and association between urinary tract infection and leukocytosis" Acta Medica, 2015, 4: 42-47.

[13] J. F. T. Akoachere, Y. Suylika, H. A. Njom, N. S. Esemu "Etiologic profile and antimicrobial susceptibility of community-acquired urinary tract infection in two Cameroonian towns" BMC Research notes, 2012; 5: 219.

[14] M. N. Mokube, J. Atashili, G. E. Halle-Ekane, G. M. Ikomey, P. M. Ndumbe "Bacteriuria amongst Pregnant Women in the Buea Health District, Cameroon: Prevalence, Predictors, Antibiotic Susceptibility Patterns and Diagnosis" PLOS Medicine, 2013; 8: 71-86.

[15] M. F. Yuyun, F. F. Angwafo, S. Koulla-Shiro, J. Zoung-Kanyi "Urinary tract infections and genitourinary abnormalities in Cameroonian men" Tropical Medicine and International Health, 2004, 9: 520-525.

[16] M. Cheesbrough "Haematological test In: District Laboratory Practice in Tropical Countries" Part 2 Second Edition, 2006, Cambridge University Press.

[17] Clinical and Laboratory Standards, Wayne PA. Performance standards for antimicrobial disk susceptibility tests. Approved standard. Clinical and Laboratory Standards Institute 2006; ninth edition Document M2-A9.

[18] National Committee on Clinical Laboratory Standards Performance Standards for Antimicrobial Susceptibility Testing: 12th supplement; M100-S12; 2002, vol 22, pp9095.

[19] G. Schmeimann, E. Kneihl, K. Gebhardt, M. M. Matejczyk, E. Hummers-pradier "The Diagnosis of Urinary Tract Infection" Dtsch Arztebi Int, 2010, 107: 361-367.

[20] B. Waite "Diagnosis and Treatment of Urinary Tract Infections in Children" American Family Physician 2011; 83: 409-415.

[21] A. L. Njunda, J. C. N. Assob, S. D. Nsagha, P. N. Fon, H. L. F. Kamga, A. F. Nkume, K. E. Tebit "Uropathogens from Diabetic Patients with Asymptomatic Bacteriuria and Urinary Tract Infections" The West London Medical Journal, 2013, 5: $7-14$.

[22] B. Kacmaz, O. Cakir, A. Aksoy, A. Biri "Evaluation of rapid urine screening tests to detect asymptomatic bacteriuria in pregnancy" Jpn J Infect Dis, 2006, 59: 261-263. 
[23] R. Leclerq "Mechanisms of Resistance to Macrolides and Lincosamides: Nature of the Resistance Elements and Their Clinical Implications” Clin. Infect. Dis., 2002, 34: 482-492.

[24] C. F. Igwe, O. O. Oduyebo, P. O. Oshun, A. O. Ettu "Prevalence and characterization of inducible clindamycin resistant Staphylococcus aureus in a tertiary institute in Lagos state” Int J Infect Trop Dis, 2015, 3 (1): 13-19.

[25] A. Arizpe, K. R. Reveles, S. D. Patel, S. L. Aitken "Updates in the management of cephalosporin-resistant Gram-negative bacteria" Curr Infect Dis Res, 2016, 18 (12): 39.

[26] E. K. Oladipo, J. K. Oloke, I. O. Omomowo, A. O. Oyeniran,
E. H. Awoyelu, S. O. Ogundele "Incidence of cephalosporin resistance among clinical isolates of Pseudomonas aeruginosa in Ibadan, South-Western Nigeria" Int J Med Biomed Res, 2015, 4 (3): 135-141.

[27] T. O. Okunola, K. O. Ajenifuja, S. O. Ogunniyi, A. O. Aboderin "Erythromycin versus amoxicillin for the management of chlamydia infection in pregnant women: a randomized controlled trial” Int J Med Biomed Res 2016; 5 (1): 35-42.

[28] G. M. Eliopoulos "In vitro activity of fluoroquinolones against gram-positive bacteria” Drugs, 1995; 49: 48-57. 